\title{
Vergleich von vier kommerziell erhältlichen Rotavirus-ELISA mittels RNA Gel-Elektrophorese. Inzidenz von Rotavirus-Diarrhöen bei Kindern und Erwachsenen
}

\author{
Comparison of Four Commercially Available Enzyme Immunoassays for Rotavirus Detec- \\ tion with RNA gel electrophoresis. \\ Incidence of Rotavirus-Diarrhea in Children and Adults
}

Th. Mertes, R. Bockmühl, M. Faulde, R. Walscheid, D. Sobe

Aus dem Fachbereich II - Medizinische Mikrobiologie des Ernst-Rodenwaldt-Institutes, Koblenz (Leiter: Dr. med. D. Sobe)

\begin{abstract}
Zusammenfassung:
In einem Zeitraum von vier Monaten (November 1988-Februar 1989) wurden 130 Stuhlproben von Säuglingen und Kindern sowie 320 Proben von männlichen Erwachsenen mit Gastroenteritis auf Anwesenheit von Rotaviren untersucht. Dafür standen vier kommerziell erhältliche Enzymimmunoassays zur Verfügung: zwei mit monoklonalen (Pathfinder-Rotavirus, Kallestad; Rotavirus ElA, Röhm Pharma) und zwei mit polyklonalen Antikörpern (Rotavirus-Enzygnost (Ag), Behring; Rotazyme II, Abbott). Die 130 Stuhlproben der Kinder wurden zusätzlich mittels RNA Gel-Elektrophorese analysiert. Die diagnostische Spezifität der zwei ELISA mit monoklonalen Antikörpern (je 98,9\%) war höher als die der zwei mit polyklonalen Antikörpern (Enzygnost $88,6 \%$, Rotazyme II 96,6\%). Die diagnostische Sensitivität des Rotavirus ElA und des Enzygnost (je 95,2\%) war etwas höher als die des Pathfinder-Rotavirus (92,9\%) und deutlich höher als die des Rotazyme II (85,7\%). Von den 130 Stuhlproben der Kinder erwiesen sich 42 (= $32 \%$ ), von den 320 Proben der Erwachsenen 9 $(=2,8 \%)$ als positiv.
\end{abstract}

Rotaviren stellen bei kindlicher Gastroenteritis das wichtigste ätiologische Agens dar, wohingegen die geringere Infektionshäufigkeit von Rotaviren bei Erwachsenen annähernd mit der von Salmonellen bzw. Campylobacter vergleichbar ist.

Schlüsselwörter:

Rotavirus - Antigen-ELISA - RNA Gel-Elektrophorese

Summary:

Between November 1988 and February 1989, 130 fecal samples from infants and children and 320 samples from male adults with gastroenteritis were tested for rotavirus by four commercially availabie enzyme immunoassays: two monoclonal (Pathfinder-Rotavirus, Kallestad; Rotavirus ElA, Röhm Pharma) and two polyclonal antibody-based assays (Rotavirus-Enzygnost (Ag), Behring, Rotazyme II, Abbott). All of the 130 fecal samples from children were examined additionally by RNA gel electrophoresis. The specificity of the two monoclonal antibody-based EIA (both $98,9 \%$ ) was superior to those of the two polyclonal antibody-based EIA (Enzygnost $88,6 \%$, Rotazyme II 96,6\%). The sensitivity of the Rotavirus EIA and Enzygnost (both 95,2\%) was similar to the Pathfinder (92,9\%) but higher than that of Rotazyme II (85,7\%). As determined, 42/130 fecal samples (= $32 \%)$ from children and $9 / 320$ of the adults' samples' $(=2,8 \%)$ were rotavirus positive.

Rotaviruses are shown to be the most important etiologic agent of 'gastroenteritis of infants and children, whilst the infection frequency of rotaviruses in adults is almost comparable with the bacterial pathogens salmonella and campylobacter.

Keywords:

Rotavirus - antigen-EIA - RNA gel electrophoresis

\section{Einleitung}

Die erste Beschreibung der Rotaviren erfolgte 1973 durch Bishop et al., die elektronenmikroskopisch virusähnliche Partikel in Duodenalschleimhaut-Epithelzellen von Kindern darstellten (1). Kurz danach konnten diese Viruspartikel auch in Stuhlaufbereitungen elektronenmikroskopisch nachgewiesen werden $(2,3)$. Da die $70 \mathrm{~nm}$ großen
Viren mit ihrem ringförmigen, scharf begrenzten äußeren Kapsid eine radähnliche Form aufwiesen, wurden sie Rotaviren genannt. Schon bald erkannte man, daß sie eine wichtige Rolle bei der schweren kindlichen Diarrhoe spielen; heutzutage besitzen diese der Familie der Reoviridae zugehörigen RNA-Viren eine weltweite Bedeutung als das wichtigste ätiologische Agens für eine im Säuglingsbzw. Kindesalter erworbene Gastroenteritis. Während 


\section{Neu für die Schilddrüsen- und Tumordiagnostik: \\ Das Berilux ${ }^{\circledR}$ System}

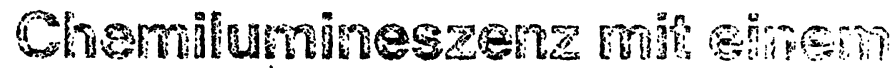

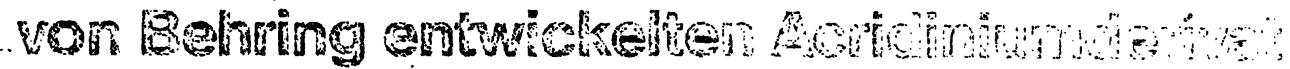

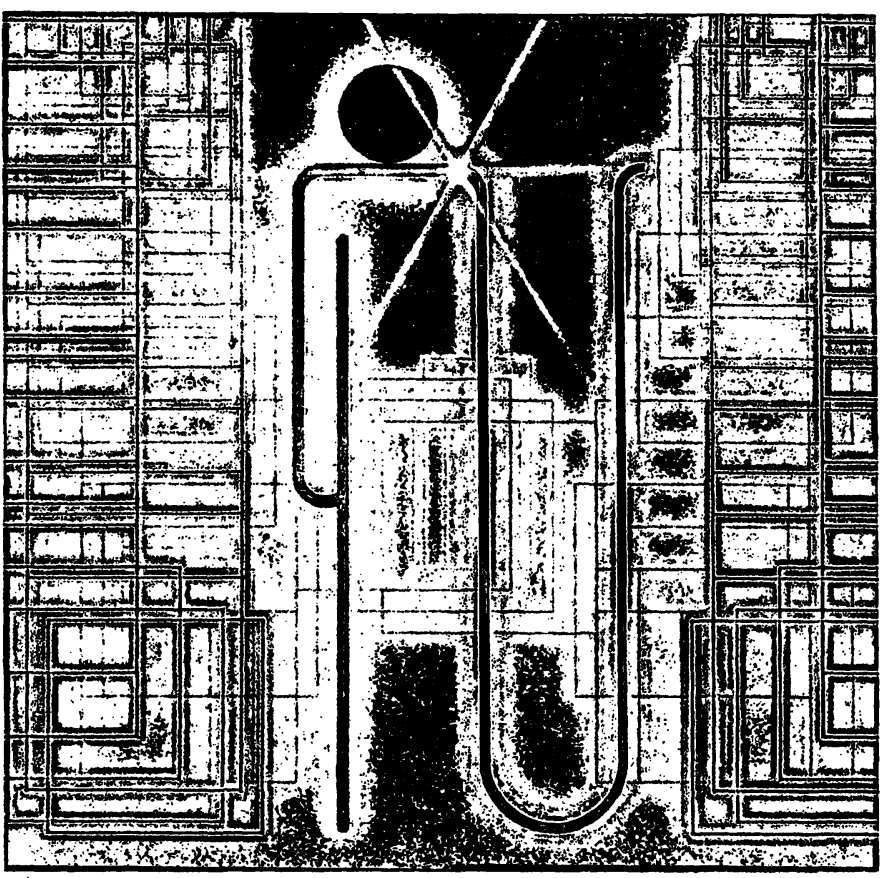

Behring bietet lhnen

mit BeriLux

ein komplettes System:

$\square$ Reagenzien

$\square$ Geräte

$\square$ Software

Service
Vorteile des BeriLux

Systems: vereinfach.te

und rationelle

Testdurchführung

Barcode-Lesung aller testspezifischen Daten

eine auf Ihre individuellen Bedürfnisse zugeschnittene Anwendersoftware

2-Punkt-Kalibrierung

$\square$ Meßzeit nur 1 Sekunde 


\section{III jedem rall zuverlassig}

Unser umfangreiches

Autoantikorpernachweis-Programm

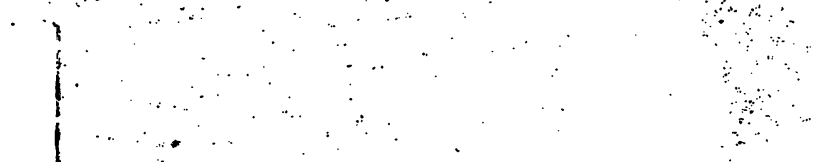

$\therefore=\therefore$

Neu in unserem Programm

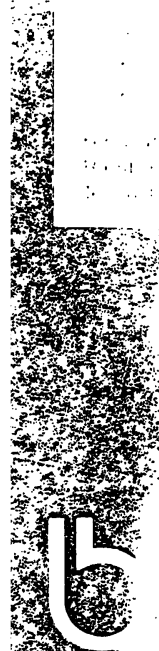

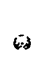

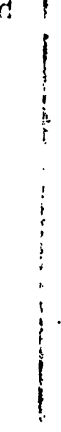


$30-50 \%(4,5,6)$ der kindlichen Diarrhöen durch Rotaviren verursacht werden, ist die Rate der Infektionen bei Erwachsenen wesentlich geringer. Die Durchseuchung im Kindesaiter ist sehr hoch und die zurückbleibende Immunität läßt bei einer Reinfektion meist nur einen subklinischen Verlauf zu. Das Hauptrisiko für eine solche Reinfektion scheint die Erkrankung von Kindern der unmittelbaren Umgebung zu sein $(7,8)$.

Bislang findet sich kein Hinweis auf Infektiosität von TierRotaviren für den Menschen; Untersuchungen mittels RNA-RNA-Hybridisierung haben deutliche Unterschiede zwischen Tier- und Mensch-Rotaviren aufgezeigt (9).

Auf dem fäkal-oralen Übertragungswege kommt es zur Infektion der Duodenalschleimhaut-Epithelzellen. Nach einer Inkubationszeit von zwei bis vier Tagen $(6,10)$ äuBert sich die Erkrankung durch Erbrechen und Diarrhoe unterschiedlichsten Schweregrades mit einer leichten Temperaturerhöhung. Das Krankheitsbild hält normalerweise drei bis sieben Tage an. Infektionen kommen in unseren Breiten über das ganze Jahr verteilt vor, die Inzidenz ist in der kälteren Jahreszeit am höchsten.
Zum Nachweis von Rotavirus-Antigen im Stuhl sind die ersten vier Krankheitstage der ideale Zeitraum; die Virusausscheidung kann bis zu 21 Tagen andauern. Ursprünglich war nur die elektronenoptische Darstellung zum Nachweis von Rotaviren in Stuhlproben möglich. Diese Untersuchungsmethode ist aufgrund der distinkten Morphologie der Erreger zwar hochspezifisch, jedoch sehr arbeitsaufwendig und bei hohen Untersuchungszahlen impraktikabel. Die Methode der Wahl zur Routinediagnostik ist inzwischen der Antigen-Nachweis durch ELISA geworden; diese immunologische Nachweismethode besitzt eine hohe diagnostische Sensitivität und kann auch mit einfachen Mitteln bei großen Untersuchungszahlen durchgeführt werden (11-19).

Ziel der Untersuchung war zum einen der Vergleich von vier kommerziell erhältlichen Antigen-ELISA anhand der RNA Gel-Elektrophorese und zum anderen die Bestimmung der Häufigkeit von Rotavirus-Infektionen bei männlichen Enteritis-Patienten, überwiegend im Alter von 18-30 Jahren.

Tab. 1: Hinweise zum Aufbau und zur Durchführung der Enzymimmunoassays

\begin{tabular}{|c|c|c|c|c|}
\hline Vorbereitung & $\begin{array}{l}\text { Enzygnost-Rotavirus (Ag) } \\
\text { (Behring) }\end{array}$ & $\begin{array}{l}\text { Rotazyme II } \\
\text { (Abbott) }\end{array}$ & $\begin{array}{l}\text { Pathfinder-Rotavirus } \\
\text { (Kallestad) }\end{array}$ & $\begin{array}{l}\text { Rotavirus EIA } \\
\text { (Röhm Pharma) }\end{array}$ \\
\hline $\begin{array}{l}\text { des Untersu- } \\
\text { chungsmate- } \\
\text { rials }\end{array}$ & $\begin{array}{l}\text { Stuhl mit Verdünnungs- } \\
\text { puffer AP } 1: 5-1: 10 \text { ver- } \\
\text { dünnen, mit Vortex mi- } \\
\text { schen, } 10 \text { min bei } 2000 \times 9 \\
\text { zentrifugieren, Überstand } \\
\text { im Test einsetzen }\end{array}$ & $\begin{array}{l}\text { Herstellung } \\
10 \% \text { eigen Verdünnung der } \\
\text { Stuhlprobe mit Verdün- } \\
\text { nungspuffer PVM Rota- } \\
\text { zyme II }\end{array}$ & $\begin{array}{l}\text { Stuhlprobe mit Verdün- } \\
\text { nungspuffer } 1: 10 \text { verdün- } \\
\text { nen }\end{array}$ & $\begin{array}{l}\text { Stuhlprobe wird mit Hilfe } \\
\text { einer im Testkit enthalte- } \\
\text { nen Transferpipette mit } \\
\text { Verdünnungspuffer } \\
(1: 20) \text { verdünnt }\end{array}$ \\
\hline
\end{tabular}

feste Phase Reaktionsvertiefungen der Mikrotiter-Platte mit SA 11-Antiserum (Kaninchen) (SA 11 = Affen-Rotavirus)

\section{Konjugat}

vom Rind stammende
NCDV-Antikörper, gekoppelt an Alkalische Phosphatase (NCDV $=$ RinderRotavirus)

\section{Durchführung} des Tests

- $150 \mu$ l Zentrifugationsüberstand in eine Reak-

\section{Kugeln beschichtet mit SA 11-Antiserum (Meer- schweinchen)}

vom Kaninchen stammende Rotavirus-Antikörper, gekoppelt an Meerrettich-Peroxidase tionsvertiefung pipettieren

- $2 \mathrm{~h}$ in feuchter Kammer bei $37^{\circ} \mathrm{C}$ inkubieren

- 3x mit Waschlösung waschen -

- Zugabe von $50 \mu$ l Konjugat

- $1 \mathrm{~h}$ bei $37^{\circ} \mathrm{C}$ inkubieren

- 3x waschen

- Zugabe von $100 \mu \mathrm{l}$ Substratlösung (p-Nitrophenyl-Phosphat)

- $30 \mathrm{~min}$ bei $37{ }^{\circ} \mathrm{C}$ im

Dunkeln inkubieren

- Reaktion mit $50 \mu / 2 N$

NAOH stoppen

- Extinktion bei $405 \mathrm{~nm}$ messen (Referenz: 492 $\mathrm{nm}$ )

- $200 \mu \mathrm{l}$ der verdünnten Stuhlprobe in die Vertiefungen der Inkubationsplatte pipettieren

- Zugabe einer Kugel in die jeweiligen Vertiefungen

$-1 \mathrm{~h}$ bei $37^{\circ} \mathrm{C}$ inkubieren

- 3x mit Waschlösung waschen

- Zugabe von $200 \mu l$ Konjugat

$-1 \mathrm{~h}$ bei $37^{\circ} \mathrm{C}$ inkubieren

- 3x waschen

- Kugeln in Teströhrchen überführen

- Zugabe von $300 \mu l$ Substratlösung (o-Phenylendiamin)

- 30 min bei RT im Dun-
Polystyrol-Röhrchen mit SA 11-Antiserum (Kaninchen)

Reaktionsvertiefungen einer Mikrotiter-Platte mit monoklonalen Antikörperń gegen VP 6 (Maus)

von der Maus stammende monoklonale Rotavirus-Antikörper, gekoppelt an Meerrettich-Peroxidase

von der Maus stammende monoklonale Rotavirus-Antikörper, gekoppelt an Meerrettich-Peroxidase keln inkubieren

- Reaktion mit $1 \mathrm{ml} 1 \mathrm{~N}$

$\mathrm{H}_{2} \mathrm{SO}_{4}$ stoppen

- Extinktion bei $492 \mathrm{~nm}$

messen (Referenz: 600 $n m$ )
- Zugabe von $100 \mu \mathrm{l}$

Konjugat in das Teströhrchen

- $300 \mu l$ Stuhlsuspension hinzupipettieren

- Röhrchen mischen und

$1 \mathrm{~h}$ bei RT inkubieren

- Röhrchen 5x mit A. d. waschen

- Zugabe von $500 \mu l$ Substratlösung (Substratpuffer + Chromogen)

- 15 min bei RT im Dunkeln inkubieren

- Reaktion mit $1 \mathrm{ml} 1 \mathrm{~N}$ $\mathrm{H}_{2} \mathrm{SO}_{4}$ stoppen

- Extinktion bei $450 \mathrm{~nm}$ messen
- Zugabe von $100 \mu$ l Probensuspension und 2 Tropfen $(=100 \mu$ ) Konjugat in die Reaktionsvertiefungen

- Platte gut mischen

- $1 \mathrm{~h}$ bei RT inkubieren

- 5x mit a. d. waschen

- Zugabe von 2 Tropfen $(100 \mu l)$ Substrat (Harnstoffperoxid) und 2 Tropfen $(=100 \mu$ l) Chromogen - 10 min bei RT im Dunkeln inkubieren

- Reaktion mit 2 Tropfen (= $100 \mu$ l) Stopp-Reagenz stoppen

- Extinktion bei $450 \mathrm{~nm}$ messen 


\section{Material und Methode}

In einer Spanne von vier Monaten (Nov. 1988-Feb. 1989) wurden 450 Stuhlproben von Enteritis-Patienten auf Rotaviren untersucht. Die 450 Patienten wurden in zwei Kollektive aufgeteilt: das erste bestand aus $\mathbf{3 2 0}$ männlichen Erwachsenen (Alter: 18-30 Jahre), das zweite aus 130 Säuglingen und Kleinkindern im Alter von vier Wochen bis drei Jahren.

Die Stuhlproben wurden mittels in den Testkits enthaltener Puffer verdünnt und anschließend nach Testanleitung auf Rotavirus-Antigenität überprüft. Jede der Proben wurde mit vier ELISA (Pathfinder-Rotavirus, Kallestad; Rotavirus ElA, Röhm Pharma; Rotavirus-Enzygnost (Ag), Behring; Rotazyme II, Abbott) und die 130 Proben des zweiten Kollektivs zusätzlich mit der RNA Gel-Elektrophorese untersucht. Außerdem wurden die Stuhlproben der erwachsenen Enteritis-Patienten bakteriologisch zum Nachweis von Salmonellen, Shigellen, Campylobacter und Yersinien aufgearbeitet.

ELISA: Die einzelnen Schritte der Testdurchführung sowie Hinweise auf den unterschiedlichen Aufbau der Testsysteme werden in Tab. 1 dargelegt. Unterschiede bestehen $u$. $a$. in der verwendeten festen Phase, an welche die Rotavirus-Antikörper fixiert sind (Reaktionsvertiefungen einer Mikrotiterplatte, Teströhrchen, Kugel) und in dem eingesetzten Konjugat (polyklonale oder monoklonale Antikörper sind an unterschiedliche Enzyme gekoppelt).

RNA Gel-Elektrophorese: Die Probenaufbereitung, die anschließende Separierung mittels PAGE (Polyacrylamidgel-Elektrophorese) sowie die Beurteilung des Bandenmusters folgten der Methode von Selb et al. (20). $20 \mu l$ des aufbereiteten Probenmaterials wurde auf $0,5 \mathrm{~mm}$ dicke $(245 \times 115 \mathrm{~mm})$ horizontale PA-Gele $(T=8 \%, C=$ $2,5 \%)^{*}$ mit einem Sammelgel $(T=5 \%, C=2,5 \%)$ appliziert. Zur mechanischen Stabilisierung wurden die verwendeten PA-Gele kovalent an eine Trägerfolie (Gel Bond Film, Serva) aufpolymerisiert. Die Elektrophorese erfolgte bei $+5^{\circ} \mathrm{C}$ und $550 \mathrm{~V}(\max .50 \mathrm{~mA})$; die Laufzeit betrug 90 min. Die RNA-Banden wurden mit einer kommerziell erhältlichen Silberfärbung (BioRAD) visualisiert.

Bei allen in der RNA-PAGE negativen Proben wurde zur Aufkonzentrierung möglicherweise in geringer Konzentration vorliegender RNA mit 10 Vol. Azeton präzipitiert, bei $5000 x g$ für 5 min zentrifugiert, der Überstand dekantiert und das Präzipitat anschließend in Probenpuffer (10\% des Ausgangsvolumens) resuspendiert. Hieran schloß sich die oben beschriebene Probenaufbereitung an.

\section{Ergebnisse}

In der RNA Gel-Elektrophorese wurden diejenigen Proben als positiv bewertet, die 11 RNA-Banden definierten Molekulargewichtes aufwiesen (20-23). Das bei einer Rotavirus-Infektion auftretende RNA-Bandenmuster ist in Abb. 1 dargestellt.

Von den 130 Stuhlproben der Säuglinge bzw. Kleinkinder wiesen 42 (= $32 \%)$ ein Rotavirus-RNA-Bandenmuster

\footnotetext{
* $\mathrm{T}=$ Gesamtkonzentration der Polymere (Acrylamid, Bis-Acrylamid)

$\mathrm{C}=\mathrm{Grad}$ der Quervernetzung
}
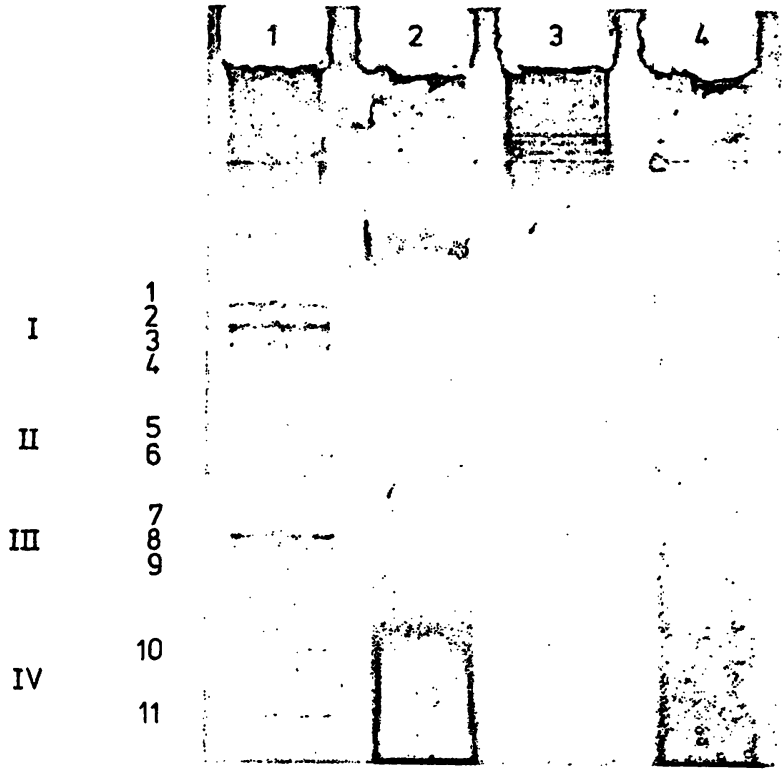

Abb. 1: Darstellung von Rotavirus-RNA nach horizontaler PAGE ( $T=8 \%, C=2,5 \%$ ) und Silberfärbung: 1) positive Probe; 2) negative Probe; 3) positive Probe nach 10facher Aufkonzentrierung; 4) negative Probe.

auf. Lediglich $33 / 42$ positive Proben wurde von allen vier ELISA erkannt. Der positive prädiktive Wert, der eine Aussage macht über die Wahrscheinlichkeit für Krankheit bei pathologischem Laborbefund, lag beim Rotavirus EIA bei $97,6 \%$ und beim Pathfinder-Rotavirus bei $97,5 \%$. Geringere positive Vorhersagewerte waren für den Rotazyme II mit 92,3\% und den Enzygnost-Rotavirus ( $\mathrm{Ag}$ ) mit $80 \%$ zu konstatieren.

Als die Wahrscheiṇlichkeit für Nicht-Krankheit bei normalem Laborwert wird der negative prädiktive Wert definiert. Hier erreichten der Rotavirus EIA 97,8\%, der Enzy-

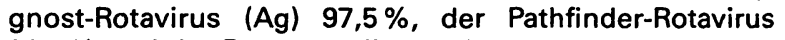
$96,7 \%$ und der Rotazyme II 93,4\%.

Die diagnostische Effizienz eines Tests beschreibt das Verhältnis der richtigen Laborergebnisse zu. der Gesamtheit aller Ergebnisse des 130 Patienten umfassenden Kollektivs. Es ergaben sich folgende Werte: Rotavirus EIA $97,7 \%$, Pathfinder-Rotavirus $96,9 \%$, Rotazyme II $93 \%$ und Enzygnost-Rotavirus (Ag) 90,8\%.

Ergebnisse hinsichtlich Sensitivität, Spezifität, des positiven und negativen prädiktiven Wertes sowie der diagnostischen Effizienz der einzelnen Testsysteme zeigt die Tab. 2.

Die elektrophoretische Untersuchung der 130 KinderStuhlproben fiel niemals positiv aus, ohne daß nicht zumindest ein Testsystem ebenfalls Rotavirus-Antigen offenbarte. Da die Schlußfolgerung gezogen werden konnte, daß die vier ELISA zusammengenommen eine sehr hohe Sensitivität aufwiesen, wurde bei dem Untersuchungsmaterial der Erwachsenen darauf verzichtet, alle Proben mittels RNA Gel-Elektrophorese zu untersuchen, sondern nur diejenigen, die in wenigstens einem Test positiv reagierten $(22 / 320=6,9 \%)$. Bei diesen 22 Stuhlproben konnte nur viermal ein Rotavirus-RNA-Bandenmuster sichtbar gemacht werden. Zwei Proben wiesen bei allen vier durchgeführten ELISA ein positives Ergebnis auf, zwei weitere bei drei Tests und eine Probe 


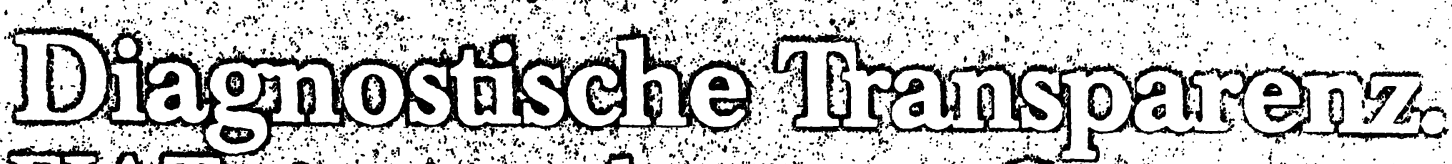

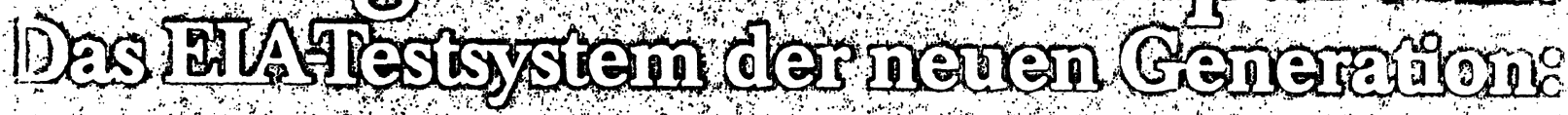
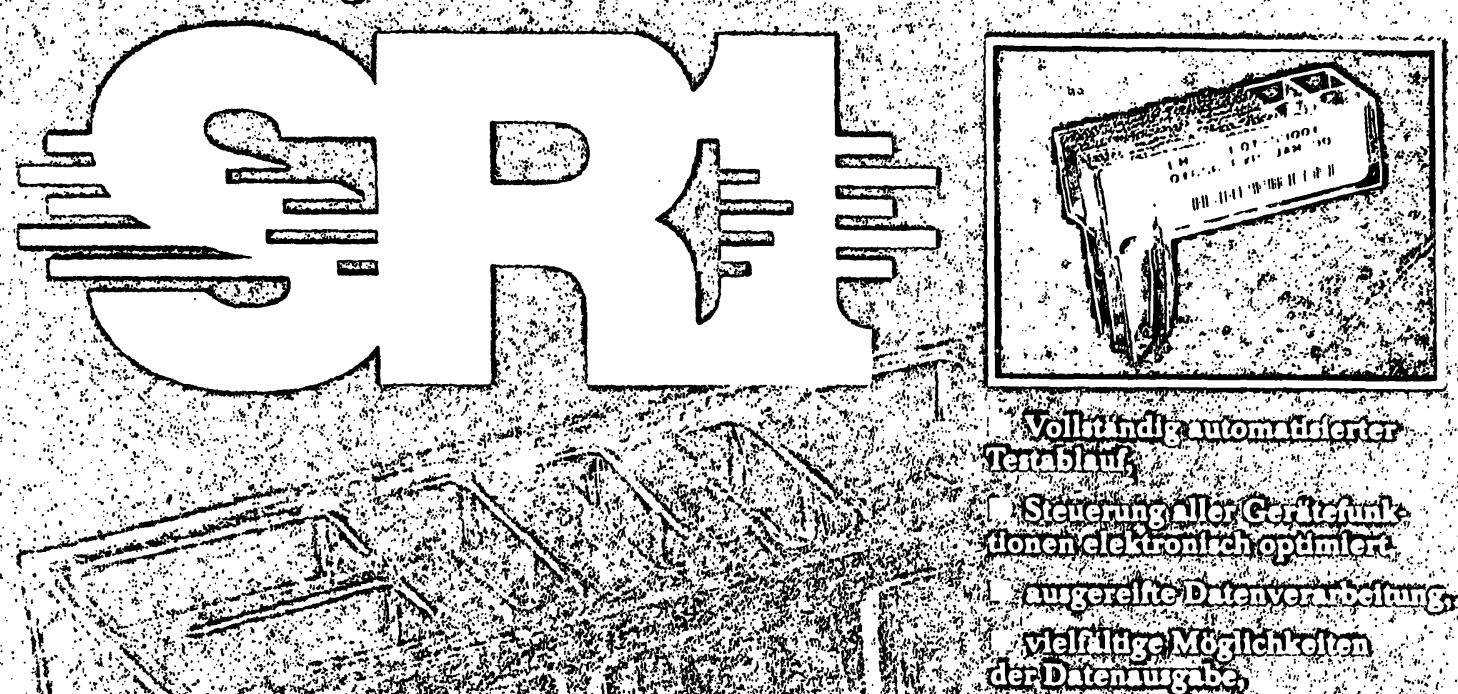

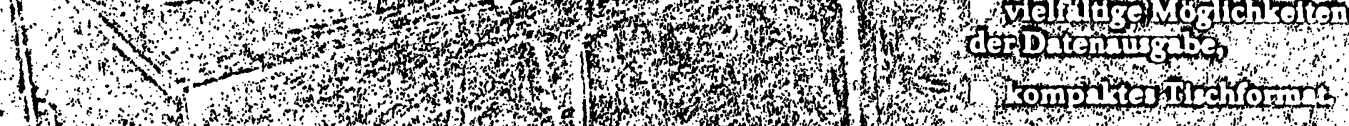

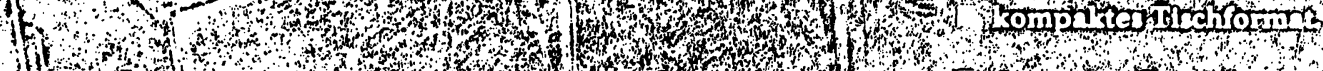

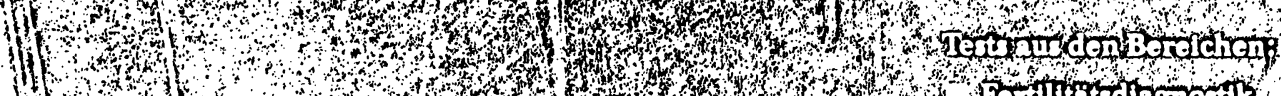
(1)

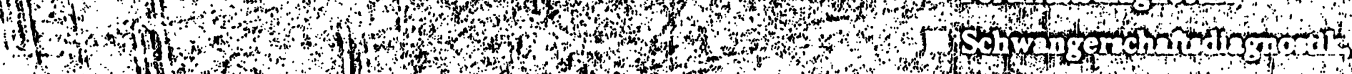

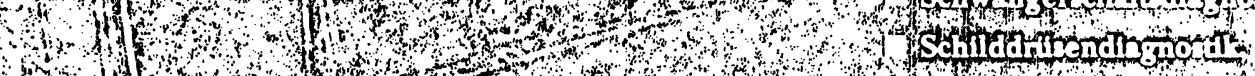
(n)

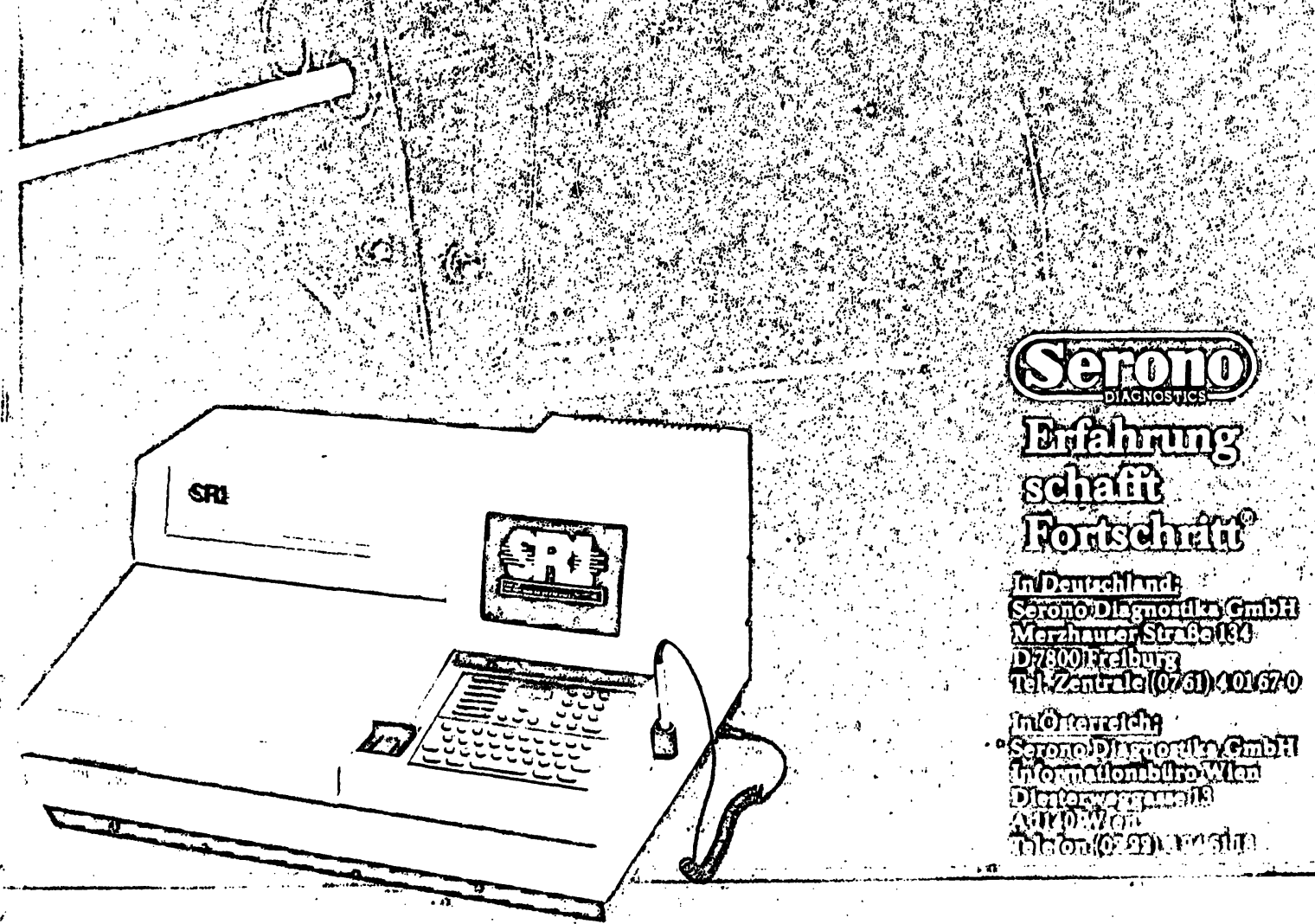




\section{Mit Du Pont für die Zukunft gerüstet}

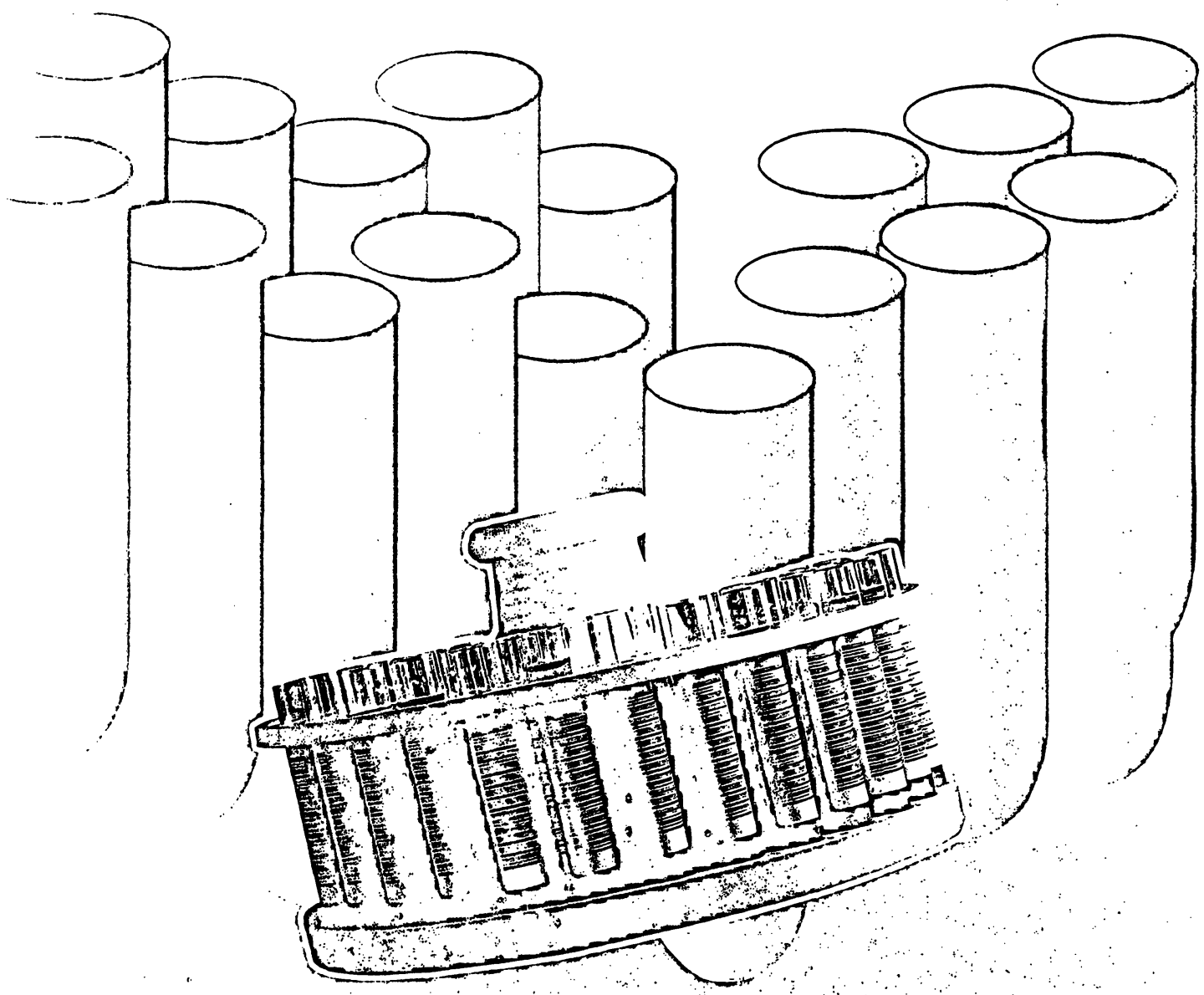

\section{Ejin Bevilrag:}

Postive Probenilotentifilkation in der Routine. dilie neue Generation des Dinension:

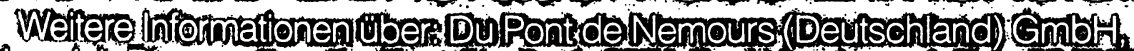

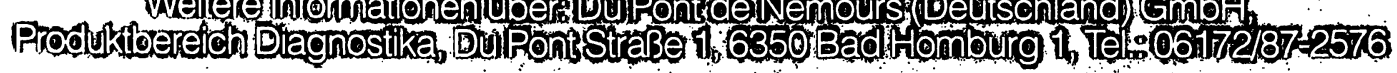

\section{(IUPOND}


Tab. 2: Ergebnisse der Testsysteme zum Nachweis von Rotavirus-Antigen in Stuhlproben $(n=130)$

\begin{tabular}{l|c|c|c|c|c|c|c|c|c}
\hline Test & $\begin{array}{c}\text { Anzahl der } \\
\text { richtig } \\
\text { positiven } \\
\text { Ergebnisse }\end{array}$ & $\begin{array}{c}\text { Anzahl der } \\
\text { falsch } \\
\text { positiven } \\
\text { Ergebnisse }\end{array}$ & $\begin{array}{c}\text { Anzahl der } \\
\text { falsch } \\
\text { negativen } \\
\text { Ergebnisse }\end{array}$ & $\begin{array}{c}\text { Anzahl der } \\
\text { richtig } \\
\text { negativen } \\
\text { Ergebnisse }\end{array}$ & $\begin{array}{c}\text { Sensitivităt } \\
(\%)\end{array}$ & $\begin{array}{c}\text { Spezifität } \\
(\%)\end{array}$ & $\begin{array}{c}\text { positiver } \\
\text { prădiktiver } \\
\text { Wert }(\%)\end{array}$ & $\begin{array}{c}\text { negativer } \\
\text { prädiktiver } \\
\text { Wert (\%) }\end{array}$ & $\begin{array}{c}\text { diagnost. } \\
\text { Effizienz } \\
(\%)\end{array}$ \\
\hline $\begin{array}{l}\text { Rotavirus ElA } \\
\text { (Röhm Pharma) }\end{array}$ & 40 & 1 & 2 & 87 & 95,2 & 98,9 & 97,6 & 97,8 & 97,7 \\
$\begin{array}{l}\text { Pathfinder- } \\
\text { Rotavirus } \\
\text { (Kallestad) }\end{array}$ & 39 & 1 & 3 & 87 & 92,9 & 98,9 & 97,5 & 96,7 \\
$\begin{array}{l}\text { Enzygnost- } \\
\text { Rotavirus (Ag) } \\
\text { (Behring) } \\
\text { Rotazyme II } \\
\text { (Abbott) }\end{array}$ & 40 & 10 & 2 & 78 & 95,2 & 88,6 & 80 & 97,5 & 90,8 \\
\hline
\end{tabular}

schließlich bei den zwei ELISA mit höchster Spezifität, ohne daß RNA nachgewiesen werden konnte. Auf eine Auswertung der Testsysteme bezüglich diagnostischer Sensitivität und Spezifität hinsichtlich der ErwachsenenStuhlproben wurde aufgrund der zu geringen Zahl an positiven Proben verzichtet.

Durch die bakteriologische Aufarbeitung der 320 Stuhlproben der erwachsenen Enteritis-Patienten konnten neunmal Salmonellen und elfmal Campylobacter jejuni angezüchtet werden. Bei keiner dieser zwanzig positiven Proben zeigte ein Rotavirus-ELISA ein positives bzw. falsch positives Ergebnis.

\section{Diskussion}

Es wurden vier kommerziell erhältliche ELISA-Testsysteme zum Nachweis von Rotavirus-Antigen mittels RNA Gel-Elektrophorese evaluiert $(n=130)$. Die konzeptionellen Unterschiede der ELISA lagen in der Verwendung verschiedener

- fester Phasen (Reaktionsvertiefungen einer Mikrotiterplatte, Polystyrol-Röhrchen, Kugel)

- an die feste Phase gekoppelter Antikörper (polyklonale Antikörper gegen Affen-Rotavirus, monoklonale Antikörper gegen VP 6)

- Konjugate (poly-/monoklonale Antikörper)(vgl. Tab. 1).

Von den 130 Stuhlproben des zweiten Patientenkollektivs konnte in 42 Fällen $(=32 \%)$ elektrophoretisch ein Rotavirus-RNA-Bandenmuster dargestellt werden.

Die höchste diagnostische Sensitivität mit je 95,2\% ergaben der Rotavirus EIA (Röhm Pharma) und der Enzygnost-Rotavirus $(\mathrm{Ag}$ ) (Behring). Für den Pathfinder-Rotavirus (Kallestad) wurde eine diagnostische Sensitivität von $92,9 \%$, für Rotazyme II (Abbott) von $85,7 \%$ ermittelt.

Während bei Enzygnost-Rotavirus (Ag) die hohe diagnostische Sensitivität eine niedrige diagnostische Spezifität $(88,6 \%)$ nach sich 209 , wiesen der gleich sensitive Rotavirus EIA (monoklonale Antikörper an der festen Phase, monoklonale Antikörper des Konjugats) und der Pathfinder-Rotavirus (monoklonale Antikörper an der festen Phase) zusätzlich eine hohe Spezifität mit je 98,9\% auf. Die geringe diagnostische Sensitivität des Rotazyme II ging mit einer Spezifität von $96,6 \%$ einher.

Von den 320 Stuhlproben der Erwachsenen waren 22 $(=6,9 \%)$ in mindestens einem Test positiv. Davon konnten jedoch lediglich vier mittels RNA Gel-Elektrophorese bestätigt werden. Von fünf Stuhlproben ohne RNA-Nachweis gaben zwei Proben in vier und zwei weitere Proben in drei ELISA eine positive Reaktion an. Die fünfte Probe war in den beiden ELISA mit hoher Spezifität positiv. Diese Kombination von negativer RNA Gel-Elektrophorese (auch nach Aufkonzentrierung der Probe mittels Azetonfällung) mit positiven ELISA-Ergebnissen war in den Stuhlproben der Kinder nicht vorgekommen. Es ist durchaus denkbar, daß die RNA Gel-Elektrophorese zwar die höchste Spezifität besitzt, daß die ELISA jedoch bei geringer Virusausscheidung sensitiver reagieren. Möglicherweise detektieren die ELISA auch bei solch niedrigen $V_{i}$ ruspartikel-Konzentrationen, in denen der Gesamtgehalt an Virus-RNA für die färberische Darstellung der elektrophoretisch aufgetrennten RNA-Banden nicht ausreicht. Die Ursache für eine geringere Viruskonzentration in einigen Stuhlproben der Erwachsenen liegt eventuell darin, daß das Untersuchungsmaterial dieser Patienten erst einige Tage nach Krankheitsbeginn zur mikrobiologischen Abklärung der Enteritis-Ätiologie ins Labor gelangt, während die Mütter von erkrankten Kleinkindern frühzeitiger den Arzt konsultieren und somit Proben mit hoher Antigenkonzentration untersucht werden können.

Betrachtet man diese fünf Proben trotz fehlender elektrophoretischer Bestätigung als positiv, so erhöht sich die Zahl der Rotavirus-Infektionen auf $9 / 320=2,8 \%$ (Erwachsenenkollektiv). Somit liegt die Inzidenz der Rotavirus-Infektionen dieser in den Wintermonaten November bis Februar durchgeführten Studie gleich hoch wie die der Salmonellen- $(9 / 320=2,8 \%)$ und ähnlich hoch wie die der Campylobacter-Infektionen $(11 / 320=3,4 \%)$. Die Anzahl der positiven Stuhlproben des Erwachsenen-Kollektivs war jedoch zu niedrig, um eine Aussage über die Sensitivität und Spezifität der Testsysteme treffen zu können.

Die Testsysteme mit monoklonalen Antikörpern (Pathfinder-Rotavirus, Rotavirus ElA) sind einfach durchzuführen und erbringen zuverlässige und infolge der kurzen Inkubationsschritte (insg. $75 \mathrm{bzw} .70 \mathrm{~min}$ ) rasche Resultate. Beim Enzygnost-Rotavirus $(\mathrm{Ag})$ muß die verdünnte Stuhlprobe durch vorherige Zentrifugation $(10 \mathrm{~min}$ bei $2000 \times g)$ geklärt und der Überstand im Test eingesetzt werden. Dies und eine insgesamt dreieinhalbstündige Inkubationszeit verzögern den Test und machen ihn umständlich. Der ebenfalls arbeitsaufwendige Rotazyme II erreichte keine zufriedenstellende diagnostische Sensitivität.

Rotaviren spielen als ätiologisches Agens einer im Säuglings- bzw Kleinkindalter erworbenen Enteritis eine entscheidende Rolle $(42 / 130=32 \%)$. Zumindest in den Wintermonaten sind Rotavirus-Infektionsraten bei Erwachsenen $(9 / 320=2,8 \%)$ vergleichbar mit denen von Salmonella spp. und Campylobacter jejuni. Diese Feststellung rechtfertigt eine routinemäßige Untersuchung auf Rotavirus-Antigen auch bei erwachsenen Enteritis-Patienten. 


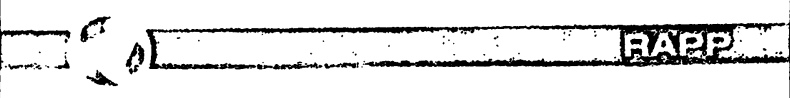 \\ RAPP GmbH -}

\section{ein Unternehmen der Stierlen-MAQUET AG}

\section{löst Ihre Reinigungsprobleme spülend!}

Wir bieten eine vielfältige Produktpalette. Unsere Anlagen sichern zukunftsorientiert Hygiene, Technik und Qualität in Krankenhäusern, Labors, Instituten, Industrie im Forschungs- und Pharmabereich sowie Apotheken.

Hohe Wirtschaftlichkeit unter Berücksichtigung des Unwweltschutzes sind selbstverständlich.

\section{Unser Programm:}

- Spezialspülautomaten für den Einsatz im Laborund medizintechnischen Bereich

- Desinfizieren, Reinigen und Trocknen - auch gem. BGA It. Gutachten und nach GMP/FDA

- Stand- und Unterbaugeräte, ein- und zweitürig, sowie Taktstraßen in verschiedenen Größen für alle Anforderungen

- Aufnahmewagen für optimale Erfassung aller anfallenden Güter, in Standard- und Sonderausführungen

- Service-Stützpunkte in ganz Deutschland durch Stierlen-MAQUET

Sind Sie interessiert und haben noch Fragen? Rufen Sie an oder schreiben Sie uns - wir beraten Sie individuell, planen und führen Versuche durch.

RAPP GmbH, Bergwerkstraße 34, D-8922 Peiting, Telefon (0 88 61) $66055-57$, Telex 59727 rapp d,

Telefax (08861) 66979

\section{Neuauflage des Standardwerkes}

\section{W. Rick}

\section{Klimische Chemie und Milkroskopie}

6., überarb. u. erweit. Aufl. 1990. Etwa 590 S. 56 Abb., davon 13 Farbtafeln, 53 Tab. Brosch. DM 29,80 ISBN 3-540-51981-5

Die vollständig neu verfaßte 6 . Auflage des erfolgreichen Lehrbuches berücksichtigt die neuesten Entwicklungen in den verschiedenen Fachdisziplinen. Es wurden eine Vielzahl neuer Untersuchungsverfahren aufgenommen und durch Herstellung klinischer Bezüge die Grundlagen für die Interpretation der Analysedaten wesentlich erweitert.

Auch die Neuauflage besticht durch die verständliche und didaktisch durchdachte Art der Darstellung, die Medizinstudenten und medizinisch-technischem Personal gleichermaßen den Einstieg in die Klinische Chemie erleichtert. Für in Klinik und Praxis tätige Ärzte ist dieses Lehrbuch ein wichtiger Ratgeber für die Indikationsstellung zur Durchführung von Analysen sowie für die Bewertung der ermittelten Daten.

Preisänderung vorbchalten

\section{Springer-Verlag Berlin Heidelberg New York London Paris Tokyo Hong Kong} Heidelberger Platz 3, D-1000 Berlin 33

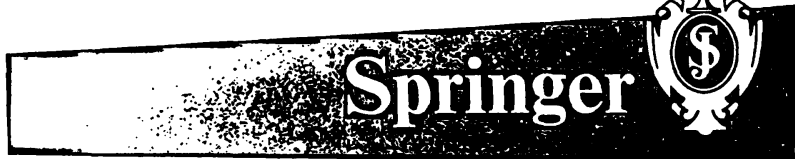

Nur zwei (Rotavirus EIA, Pathfinder-Rotavirus) der vier ge. testeten ELISA-Verfahren erreichten in der vorliegenden Untersuchung im Vergleich mit der RNA Gel-Elektropho. rese gute Ergebnisse bezüglich der diagnostischen Sensi. tivität und Spezifität.

Schriftum:

1. BISHOP, R. F., DAVIOSON, G. P., HOLMES, I. H., RUCK, B. J.: Virus particles in apith lial cells of duodenal mucosa from children with viral gastroenteritis. Lancer 2 (1973).

2. BISHOP, R. F., DAVIDSON, G. P., HOLMES, I. H., Ruck, B. J.: Detection of a new vinu by electron microscopy of fecal extracts from children with acute gastroenteritis. Lar cet 1, 149-151 (1974)

3. FLEWETT, T. H., BRYDEN, A. S., DAVIES, H.: Virus particles in gastroenteritis. Lancel 2. 1497 (1973).

4. BRANDT, C. D., KIM, H. W., RODRIGUEZ, W. J., ARROBIO, J. O., JEFFRIES, B. Pediatric viral gastroenteritis during eight years of study. J. Clin. Microbiol. 18, 71-78 (1983).

5. CUKOR, G., BLACKLOW, N. R.: HUman viral gastroenteritis. Mikrobiol. Rev, 48. $157-179(1984)$

6. DAVIDSON, G. P., BISHOP, R. F., TOWNLEE, R. R., HOLMES, I. H, RUCK, B. J. ImPO tance of a new virus in acute sporadic enteritis in children. Lancet 1, 242-245 (1975). 7. KIM, H. W., BRANDT, C. D., KAPIKIAN, A. Z., WYATT, R. G., et al.: Human reovirus like agent (HRVLA) infection: occurrence in adult contacts of pediatric patients with gastroenteritis. JAMA 238, 404-407 (1977).

8. TALLET, S., MacKENZIE, C., MIDDLETON, P., KERZNER, B., HAMILTON, R.: Clinical, laboratory and epidemiological features of viral gastroenteritis in infants and children. Pediatrics 60, 217-222 (1977).

9. FLORES, J., SERENO, M., LAI, C. J., BOEGGEMAN, E., PEREZ, l., et al.: Use of single-stranded rotavirus RNA transcripts for the diagnosis of rotavirus infection, the study of genetic diversity among rotaviruses, and the molecular cloning of rotavinus genes. In: Double-stranded RNA Viruses, edited by R. W. Compons and D. H. L genes. In: Double-stranded RNA Viruses, edite

10. KAPIKIAN, A. Z., WYATT, R. G., LEVINE, M. M., YOLKEN, R. H., VAN KIRK, D. H et al.: Oral administrat of human rotavirus to volunteers: induction of iliness and correlates of resistance J. Infec. Dis. 147, 95-106 (1983).

11. MORINET, F., FERCHAL, F., COLIMON, R., PEROL, Y.: Comparison of six methods for detecting human rotavirus in stools. Eur. J. Clin. Microbiol. 3, 136-140 (1984).

12. SAMBOURG, M., GOUDEAU, A., COURANT, C., PINON, G., DENIS, F.: Direct appraisal of latex agglutination testing, a convenient alternative to enzyme immunoassay for detection of rotavirus in childhood gastroenteritis, by comparison of two enzyme im munoassays and two latex tests. J. Clin. Microbiol. 21, 622-625 (1985)

13. CHERNESKY, M., CASTRICIANO, S., MAHONY, J., DeLONG, D.: Examination of the Rotazyme II Enzyme immunoassay for the diagnosis of rotavirus gastroenteritis Rotazyme II Enzyme immunoassay

J. Clin. Microbiol. 22, 462-464 (1985). munoassays for the diagnosis of rotavirus gastroenteritis during the course of infec tion. J. Clin. Microbiol. 22, 693-698 (1985)

15. KNISLEY, C. V., BEDNARZ-PRASHAD, A. J., PICKERING, L. K.: Detection of rotavirus in stool specimens with monoclonal and polyclonal antibody-based assay systems. J. Clin. Microbiol. 23, 897-900 (1986).

16. CROMIEN, J. L, HIMMELREICH, C. A., GLASS, R. I., STORCH, G.A.: Evaluation of new commercial enzyme immunoassay for rotavirus detection. J. Clin. Microbiol. 12 new commercial ent

17. THOMAS, E. E. PUTERMAN, M. L, KAWANO, E. CURRAN, M. Evaluation of seven immunoassays for detection of rotavirus in pediatric stool samples. J. Clin. Microbiol. 26, 1189-1193 (1988).

18. DENNEHY, P. H., GAUNTLETT, D. R., TENTE, W. E.: Comparison of nine commercia immunoassays for the detection of rotavirus in fecal specimens. J. Clin. Microbiol. 26 1630-1634 (1988).

19. GILCHRIST, M. J. R., BRETL, T. S., MOULTNEY, K., KNOWLTON, P. R., WARD, R. L.: Comparison of seven kits for detection of rotavirus in fecal specimens with a sensitive specific enzyme immunoassay. Diagn. Microbiol. Infec. Dis. 8, 221-228 (1988). 20. SELB, B BAUMEISTER, H. G MAAS, G. DOERR, H. W. Detection of human rot virus by nucleic acid analysis in comparison to enzyme-linked immunassay and elec tron microscopy. Eur. J. Clin. Microbiol. 4, 41-45 (1985).

21. DOLAN, K T TWIST, E. M. HORTON-SLIGHT, P., FORRER, C., BELL, L. M., et al: Epidemiology of rotavirus electropherotypes determined by a simplified diagnostic echnique with RNA analysis. J. Clin. Microbiol. 26, 753-758 (1985).

22. CLARK, J. D., HILL, S. M., PHILLIPS, A. D.: Investigation of hospital-acquired rotavrus gastroenteritis using RNA-electrophoresis. J. Med. Virol. 26, 289-299 (1988). 23. Arens, A., Swierkosz, E. M.: Detection of rotavirus by Hybridization with a nonradioactice synthetic DNA probe and comparison with commercial enzyme immunoas. says and silver-stained polyacrylamide gels. J. Clin. Microbiol. 27, 1277-1279 (1989).

Anschrift der Autoren:

Dr. med. Thomas Mertes Ernst-Rodenwaldt-Institut Viktoriastraße $11-13$ 5400 Koblenz

Ralf Bockmühl

Dr. rer. nat. Michael Faulde Dr. med. Rüdiger Walscheid Dr. med. Dirck Sobe

Ernst-Rodenwaldt-Institut

Viktoriastraße 11-13

5400 Koblenz 